\title{
PULSED-SOURCE INTERFEROMETRY FOR CHARACTERIZATION OF RESONANT MICROMACHINED STRUCTURES
}

\author{
Roman C. Gutierrez, Kirill V. Shcheglov, and Tony K. Tang \\ Jet Propulsion Laboratory, California Institute of Technology \\ Pasadena, CA 91109-8099
}

\begin{abstract}
We present a new microscopy tool for quantitative measurement of the resonant motion of microstructures. The new instrument uses optical interferometry to generate a threedimensional surface topographic map of a vibrating micromachined resonator in vacuum. By strobing the light source, the image of the moving device is frozen at an arbitrary position in its vibration cycle. By varying the phase between the excitation signal and the pulsing of the light source, the out of plane motion of the resonant device can be measured with a resolution of less than $0.5 \mathrm{~nm}$.
\end{abstract}

\section{INTRODUCTION}

Resonant micromachined structures are used for many applications in which the frequency, the damping and the coupling of the resonant modes of the structure is used to measure acceleration, pressure, rotation rate, and other physical and chemical phenomena. To characterize these devices it is necessary to understand the dynamic properties of the micromachined structures. Conventional microscopy tools accurately measure the static properties of MEMS devices, but dynamic properties are not available with these techniques. This paper describes a new instrument that measures dynamic properties of MEMS structures.

\section{PRINCIPLE OF OPERATION}

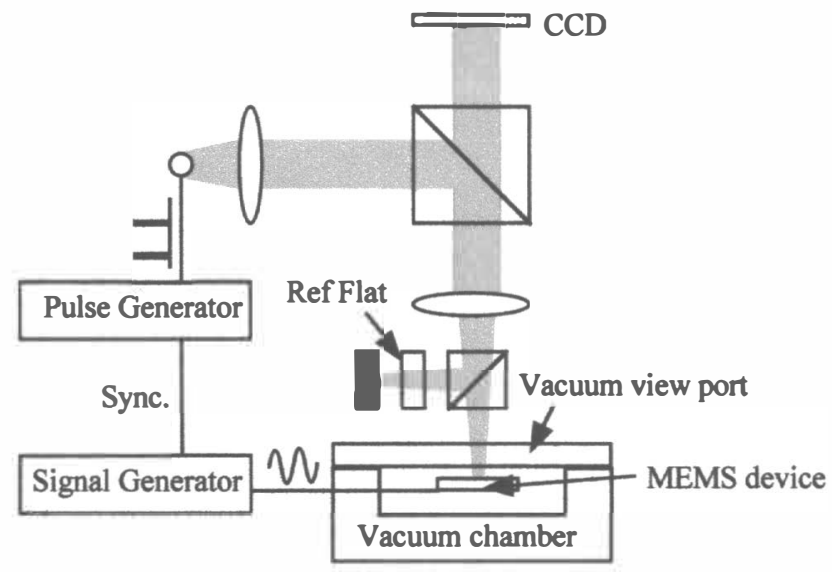

Figure 1. Schematic of the modified instrument.

Optical interferometry uses the interference of light to accurately measure distances. When two light beams of equal intensity are superimposed, the intensity of the light in the overlapping region varies between zero and twice the total intensity of the two beams. The pattern generated is usually referred to as a fringe pattern. The intensity at any point of the fringe pattern is a measure of the relative phase shift between the two beams. For metrology, the path that one of the beams travels is fixed, and the fringe pattern is a measure of the distance that the other beam traversed. The WYKO RST Plus optical profiler, a commercially available instrument, utilizes this principle to accurately measure surface topography of static structures [1, 2]. This instrument was modified to enable measurement of resonating structures. A diagram of the new instrument is shown in Figure 1. The halogen light bulb used in the original instrument has been replaced with an LED to enable rapid pulsing of the light source. The LED is driven by a variable-width pulse generator synchronized to a signal generator which excites the structure. Since many micromachined structures do not vibrate at atmospheric pressure, the structure is placed in a small vacuum chamber. A matched optical flat is used in the reference arm of the interferometer to allow interferometry through the viewport glass using a broad spectrum source.

Figure 2(a) shows an interferogram of a micromachined cloverleaf structure resonance mode at $7.767 \mathrm{kHz}$ using continuous LED illumination. A significant blurring of fringes is observed, and the node-lines, where the motion is much less than the wavelength of the source, are clearly visible as areas with highcontrast fringes. Upon switching to pulsed-source illurnination, high-contrast interference fringes are recovered over the whole structure as seen in Fig. 2(b). Figure 2(c) shows the structure under the same conditions as 2(b), except it has been tilted to accentuate the symmetry of the mode.
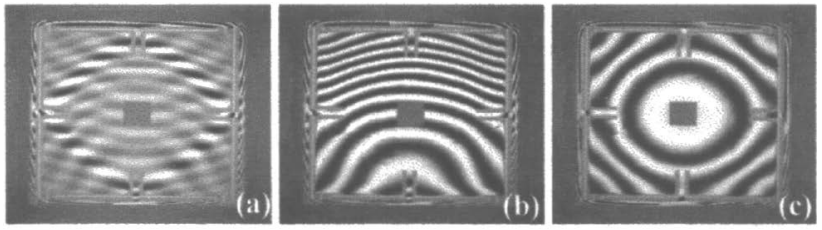

Figure 2. Fringe pattern of the vibrating cloverleaf structure with (a) continuous illumination, (b), (c) pulsed illumination.

The WYKO instrument uses a piezo vertical translation stage and a software package called WYKO Vision ${ }^{\mathrm{TM}}$ to interpret the fringe patterns and generate a high resolution surface profile. Figure 3 shows a three dimensional rendering of the cloverleaf structure modeshape corresponding to the fringe patterns in figure 2 .

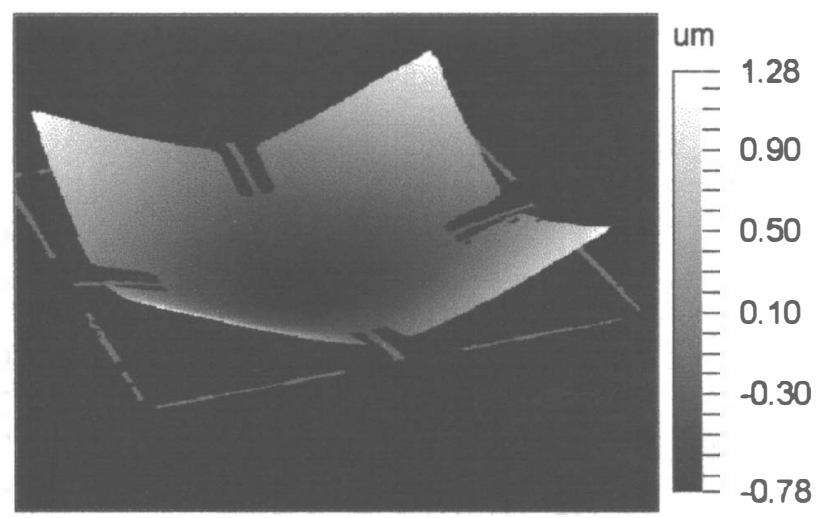

Figure 3. Modeshape of the $7.767 \mathrm{kHz}$ mode. 
The modeshape is obtained by subtracting the two surface profiles at the maximum deflection points. (Note that one modeshape is insufficient when measuring the motion due to two nearly degenerate modes.)

\section{EXPERIMENTAL RESULTS}

This instrument has been used to characterize a number of micromachined devices. Here, we will show the results for a clover leaf structure used for a microgyro [3] and a vacuum sensor [4], and a deformable mirror for adaptive optics [5].

Using this instrument, we can determine all resonance modeshapes and frequencies, as well as Q's. These measurements are used to determine mechanical parameters such as membrane tension (in the case of the deformable mirror) and spring constants, characterize asymmetry, determine location of cracks and defects, and to aid analytical modeling.

The resonance frequencies are found by tuning the excitation frequency with the LED in pulsed mode. As the frequency approaches resonance the fringe pattern changes. The $Q$ is then measured by measuring the width of the resonance peak.

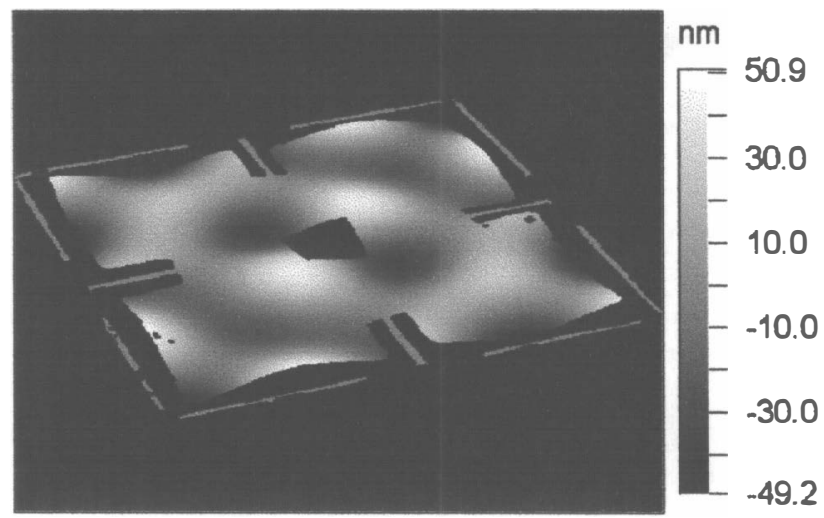

Figure 4. Modeshape of the $102.270 \mathrm{kHz}$ resonance.

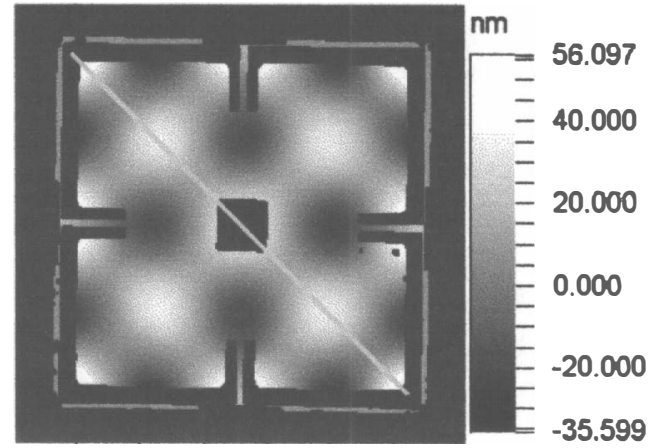

Figure 5. Modeshape of the $105.615 \mathrm{kHz}$ resonance.

For the cloverleaf structure, we measured all modeshapes and frequencies for resonances up to $200 \mathrm{kHz}$. Each clover leaf in this structure is $2 \mathrm{~mm}$ on each side and 26 microns thick. The springs are about 100 microns wide. Figure 4 shows the modeshape of a resonance found at $102.270 \mathrm{kHz}$. The amplitude of the mode is only $50 \mathrm{~nm}$ peak-peak, but the instrument can easily resolve the motion. Quantitative information can be obtained as well, which is particularly useful when comparing identical devices. Figure 5 shows a different modeshape at $105.615 \mathrm{kHz}$. Figure 6 shows a cross-sectional profile along the diagonal of the cloverleaf. This plot reveals slight asymmetries in the mode which are probably related to variations in the thickness of the epilayer used in the bulk micromachining process.

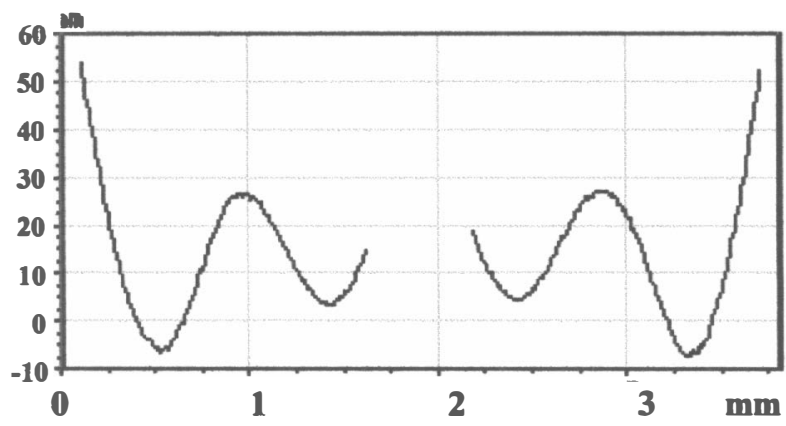

Figure 6. Cross-sectional profile of the $105.615 \mathrm{kHz}$ mode along the diagonal shown in Figure 5. Vertical scale is in nanometers.

For the deformable mirror structure, modeshapes were measured up to $200 \mathrm{kHz}$ as well. The mirror is made out of a $1 \mathrm{~cm}$ diameter Silicon Nitride membrane 0.5 microns thick with metal deposited on both sides. Table 1 shows several representative modeshapes that demonstrate the capability of the intrument. For the lower frequency modes, these measurements match theory relatively well. The surface is defined by circle polynomials where the radial variations are given by Bessel functions and the circumferential variations are sinusoidal functions. But as the fequency increases, the asymmetry in the membrane is more evident. This asymmetry is thought to come from a variation in the membrane tension. Note that all modes seem to be "locked" along the diagonal from the upper left corner to the lower right.

\section{INSTRUMENT PERFORMANCE}

The minimum resolvable out-of-plane motion was determined by taking a difference of measurements at the same position in the vibration cycle. The resulting RMS value integrated over the whole surface was $0.5 \mathrm{~nm}$. This is the same as the resolution of the unmodified instrument for stationary structures.

When the motion of the structure is large, a small illumination duty cycle is required to maintain high contrast fringes. To maintain reasonable contrast, we have found that the motion in the duration of the illumination pulse should not exceed $50 \mathrm{~nm}$ or 10 $\%$ of the maximum structure motion, whichever is less. The LED's we used have a modulation bandwidth of $3 \mathrm{MHz}$. This puts a limit of about $300 \mathrm{kHz}$ on the maximum frequency of motion that the instrument can measure. The maximum amplitude of motion resolvable at $300 \mathrm{kHz}$ is about $600 \mathrm{~nm}$ for the LED presently used. These limitations can be overcome by using a semiconductor laser diode. The large modulation bandwidth of laser diodes would allow imaging of moving structures at frequencies up to about 100 $\mathrm{MHz}$, which should be sufficient for any micromachined structure.

Presently, the lateral resolution is limited to 10 microns, since the lowest magnification objective was the only one that we fitted with the compensation optical flat required to measure inside the vacuum chamber. In principle, a higher magnification objective can be modified to match the latteral resolution of commercial optical microscopes ( $<1$ micron). Since the structure is in vacuum, an important consideration is the working distance of the objective. With the current vacuum system, which has a $6 \mathrm{~mm}$ thick viewport, the working distance of the objective must be larger than $1 \mathrm{~cm}$. 


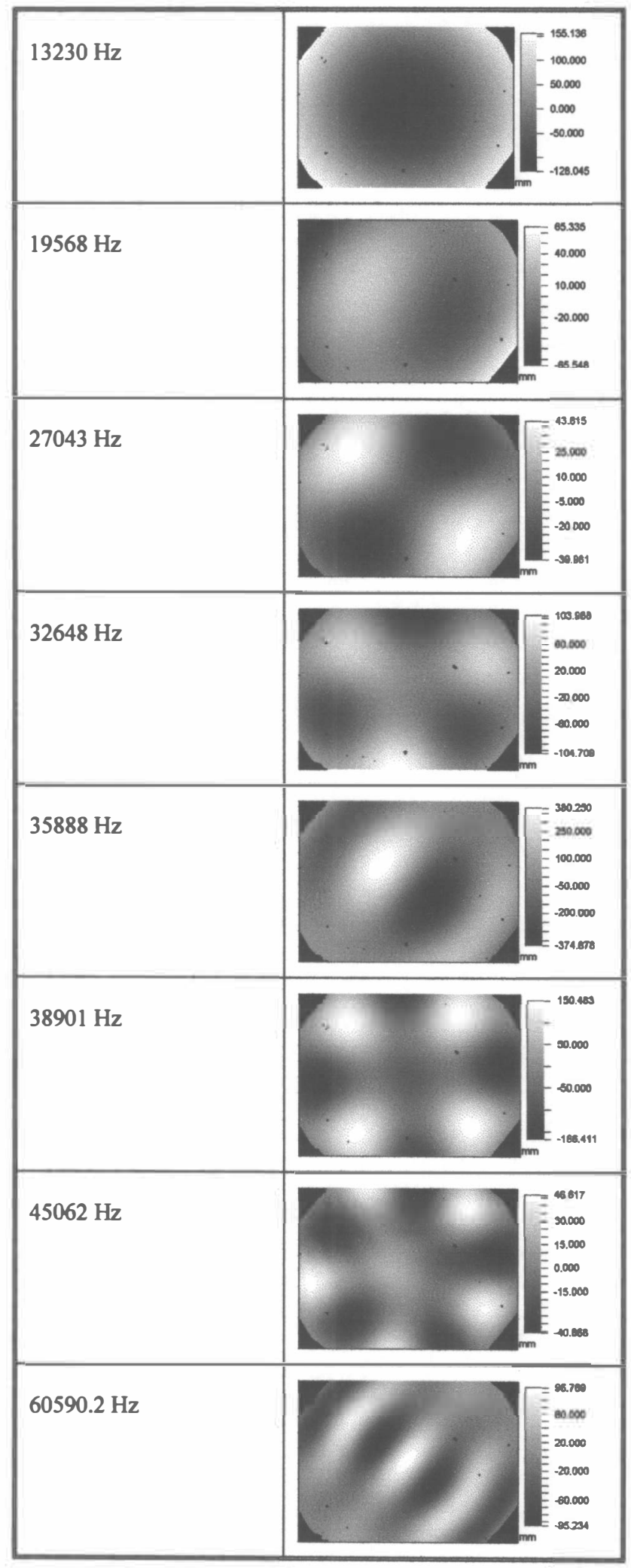

Table 1. Selected modeshapes of the deformable mirror membrane

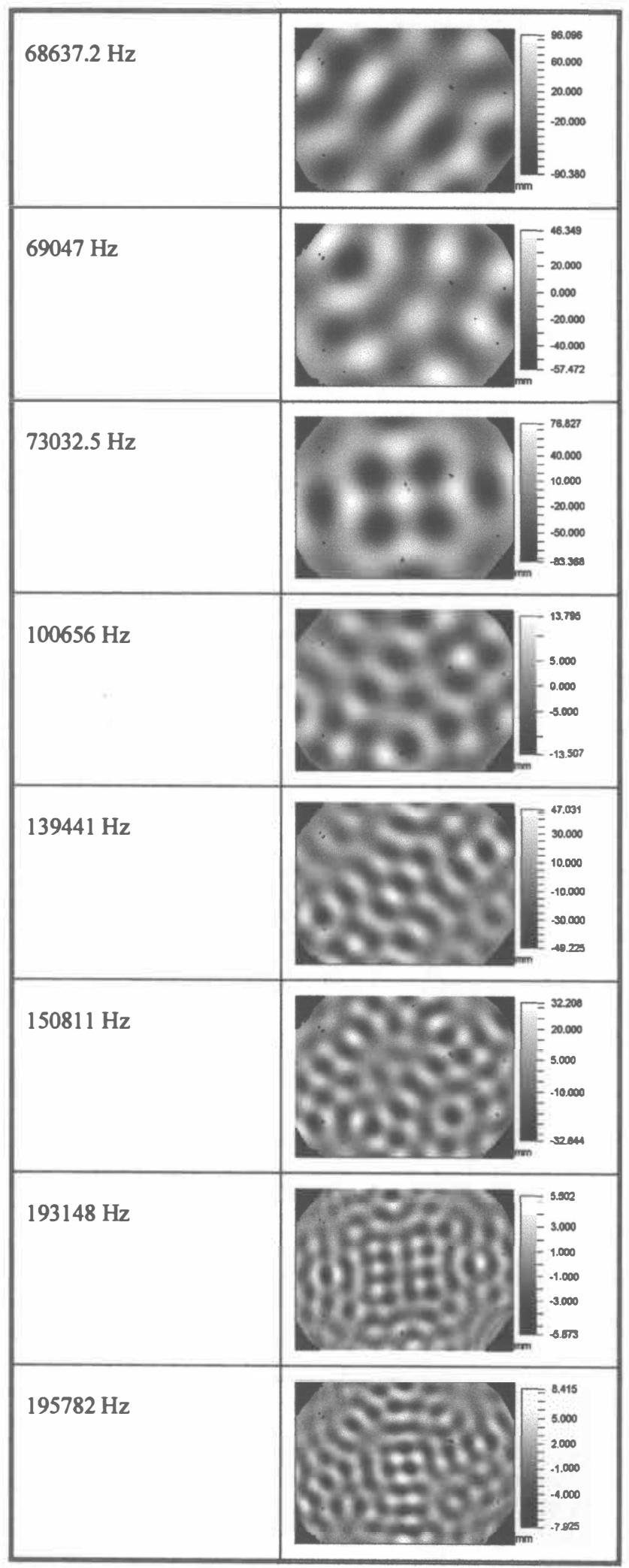




\section{APPLICATIONS}

Potential applications of the pulsed-source imaging interferometer are very broad. While it was initially used to characterize the motion of microgyroscopes, the information this instrument provides is critical to characterizing mechanical properties for a wide array of MEMS devices. In the study of MEMS reliability, for instance, an accurate knowledge of the motion of the structure over long periods of time can help solve some of the most fundamental questions related to the lifetime and failure mechanisms of micromachined devices.

With a solid state laser light source the pulsed-source imaging interferometer should enable measurements of high frequency micro-mechanical resonators, such as acousto-optic modulators, SAW filters, quartz crystal oscillators, and micromachined RF resonators.

The inventors envision an eventual marriage of the instrument with FEA modeling software. Numerical modeling generates a prediction of resonance frequencies and modeshapes from three dimensional structural data. Comparing the predictions with the experimental data, an iterative process to match the two should result in a more accurate model of real micromachined structures. This will enable a much faster device development cycle.

\section{CONCLUSION}

This paper describes a pulsed-source imaging interferometer which was constructed by modifying a commercial optical profiler manufactured by the WYKO corporation. The instrument is used to measure resonance frequencies, modeshapes, and Q's of MEMS structures. The instrument measures motion with a resolution of $0.5 \mathrm{~nm}$ RMS which is the same as the profile resolution of the unmodified instrument for stationary structures. This instrument can be used to characterize a broad spectrum of MEMS devices adding important new capabilities to the MEMS research community.

A patent for this new technology has been filed by the Califomia Institute of Technology.

\section{ACKNOWLEDGMENTS}

The work described in this paper was performed by the Center for Space Microelectronics Technology, Jet Propulsion Laboratory, California Institute of Technology, under contract with the National Aeronautics and Space Administration.

\section{REFERENCES}

1. P.J. Caber, "Interferometric profiler for rough surfaces", Appl. Opt. 32, 3438-3441 (1993).

2. D.K. Cohen, P.J. Caber, and C.P. Brophy, "Rough Surface Profiler and Method", United States Patent No. 5,133,601 (1992).

3. T.K.Tang, R.C.Gutierrez, J. Wilcox, C. Stell, V. Vorperian, R. Calvet, W. Li, I. Charkaborty, R. Bartman, W. Kaiser, "Silicon Bulk Micromachined Vibratory Gyroscope", Tech Digest, SolidState Sensor and Actuator Workshop, Hilton Head, S.C. pp.288293, June 1996.

4. Roman C. Gutierrez, Tony K. Tang, Christopher B. Stell, Vatche Vorperian, and Kirill V. Shcheglov, "Bulk Micromachined Vacuum Sensor", the Ninth International Conference on SolidState Sensors and Actuators, Transducers '97, Chicago, IL, 1997.

5. L.M. Miller, M.A. Agronin, R.K. Bartman, W.J. Kaiser, T.W. Kenny, and E.C. Vote, "A Micromachined Deformable Mirror for
Adaptive Optics Applications", 40th National Symposium of the American Vacuum Society, Orlando, FL, 1993. 